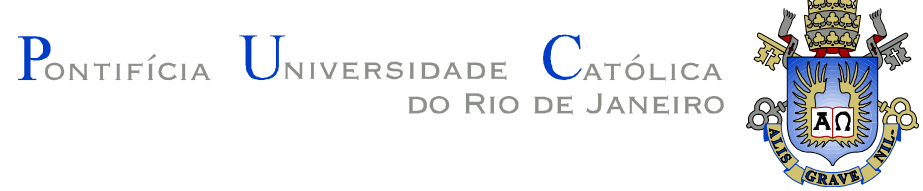

Fabrício Broseghini Barcelos

Modelagem e Previsão do Volume de Óleo Recuperável: Metodologia e Aplicação em Bacias Brasileiras

Tese de Doutorado

Tese apresentada ao Programa de Pós-Graduação em Engenharia de Produção da PUC/Rio como requisito parcial para obtenção do título de Doutor em Engenharia de Produção.

Orientador: Sílvio Hamacher

VOLUME I

Rio de Janeiro, Agosto de 2006. 
Fabrício Broseghini Barcelos

\section{Modelagem e Previsão do Volume de Óleo Recuperável: Metodologia e Aplicação em Bacias Brasileiras}

Tese apresentada como requisito parcial para obtenção do grau de Doutor pelo Programa de Pósgraduação em Engenharia de Produção da PUCRio. Aprovada pela Comissão Examinadora abaixo assinada.

Prof. Sílvio Hamacher

Orientador

Departamento de Engenharia Industrial - PUC/Rio

Prof. Cláudio Bettini

Departamento de Geologia - UFRJ

Prof. Leonardo Junqueira Lustosa

Departamento de Engenharia Industrial - PUC/Rio

Reneu Rodrigues da Silva

Empresa de Pesquisa Energética - EPE

Prof. Virgílio José Martins Ferreira Filho Departamento de Engenharia Industrial - UFRJ

Prof. José Eugênio Leal

Coordenador Setorial do Centro

Técnico Científico - PUC-Rio

Rio de Janeiro, 28 de Agosto de 2006. 
Todos os direitos reservados. É proibida a reprodução total ou parcial do trabalho sem autorização da universidade, do autor e do orientador.

\section{Fabrício Broseghini Barcelos}

Graduou-se em Engenharia Civil na UFES (Universidade Federal do Espírito Santo) em 1999. Obteve o Mestrado em Engenharia de Produção na PUC/Rio em 2002 cuja dissertação rendeu o prêmio de terceiro colocado no Development Prize Competition na XV Triennial Conference IFORS (International Federation of Operation Research Societies) sediado em Edimburgo. Publicou artigos em revistas nacionais e internacionais na área de Pesquisa Operacional. Participou de um projeto da Petrobras em parceria com a PUC/Rio e a UFRJ. Bolsista de intercâmbio universitário na Universidade de Tulsa (Oklahoma/USA) durante o ano de 2005. Atualmente trabalha como Engenheiro Pleno na Diretoria de Logística da Companhia Vale do Rio Doce.

Ficha Catalográfica

Barcelos, Fabrício Broseghini

Modelagem e previsão do volume de óleo recuperável: metodologia e aplicação em bacias brasileiras / Fabrício Broseghini Barcelos ; orientador: Sílvio Hamacher. - 2006.

262 f. : il. ; $30 \mathrm{~cm}$

Tese (doutorado em Engenharia Industrial)Pontifícia Universidade Católica do Rio de Janeiro, Rio de Janeiro, 2006.

Inclui bibliografia

1. Engenharia Industrial - Teses. 2. Evolução das descobertas. 3. Volume do óleo recuperável. 4. Bacia petrolífera. 5. Previsão. I. Hamacher, Sílvio. II. Pontifícia Universidade Católica do Rio de Janeiro. Departamento de Engenharia Industrial. III. Título. 


\section{Agradecimentos}

Ao professor Sílvio Hamacher, orientador da tese, pela oportunidade, receptividade, críticas e conhecimento adquirido.

Aos professores Cláudio Bettini, Leonardo Junqueira Lustosa, Reneu Rodrigues da Silva e Virgílio José Martins Ferreira Filho por terem aceitado o convite para participar da banca examinadora e por suas contribuições ao trabalho.

À Petrobras, em especial aos engenheiros da E\&P-ENGP Juan Antonio Molina, Fábio de Oliveira Andrade Jr. e Olavo Colela pelo fornecimento dos conjuntos de dados e contribuições ao trabalho.

À CAPES pela bolsa de estudo concedida ao longo desses anos.

Aos colegas e funcionários do DEI pela atenção e amizade.

Aos meus familiares que sempre contribuíram de alguma forma para este trabalho. Agradeço em especial à minha mãe, ao meu pai e à Suzani pelo apoio e incentivo, fundamental para mais esta vitória. 


\section{Resumo}

Barcelos, Fabrício Broseghini; Hamacher, Silvio. Modelagem e Previsão do Volume de Óleo Recuperável: Metodologia e Aplicação em Bacias Brasileiras. Rio de Janeiro, 2006. 262p. Tese de Doutorado Departamento de Engenharia Industrial, Pontifícia Universidade Católica do Rio de Janeiro.

A presente tese apresenta e discute metodologias para prever o volume de óleo recuperável em bacias petrolíferas e explicar a evolução do processo de descoberta. A evolução do processo de descoberta é modelada pelo produto de duas funções matemáticas de tendências opostas: a função seleção de controle, crescente, que representa o grau de conhecimento e informação adquiridos na região de exploração, e a função seleção de condições, decrescente, indicando que a condição de exploração piora em conseqüência da depleção da área considerada. São propostas três novas metodologias que utilizam funções de controle nãolineares para explicar a influência do progresso tecnológico no acréscimo dos volumes recuperáveis. Além disso, utiliza-se o esforço exploratório, representado pela quantidade de poços já perfurados, como variável explicativa para as funções de controle e condição. As metodologias acima mencionadas foram testadas utilizando dados históricos referentes a cinco bacias petrolíferas. Após avaliar a capacidade explicativa dos modelos através do ajuste aos dados históricos, foram feitas previsões (out of sample) para um horizonte de 3 e 10 anos com o objetivo de avaliar a capacidade preditiva. Os testes feitos com dados de quatro diferentes bacias indicam que o uso do esforço de perfuração como variável explicativa pode melhorar a previsão a longo prazo. A análise nos resíduos dos modelos propostos indica que os modelos têm boa capacidade explicativa, pois capturaram a informação contida nos dados descrevendo satisfatoriamente o processo de evolução de descobertas nas séries observadas.

\section{Palavras-chave}

Evolução das Descobertas, Volume de Óleo Recuperável, Bacia Petrolífera, Previsão. 


\section{Abstract}

Barcelos, Fabrício Broseghini; Hamacher, Silvio. Modeling and Forecast of the Recoverable Oil Volume: Methodology and Application in Brazilian Basins. Rio de Janeiro, 2006. 262p. D.Sc. Dissertation Industrial Engineering Department, Catholic University of Rio de Janeiro.

This dissertation presents methodologies to forecast the recoverable oil volume in sedimenary basins and to explain the evolution of the discovery process. The evolution of the discovery process is modeled as the product of two mathematical functions of opposing trends, namely, the control function, increasing, which represents the degree of knowledge and information acquired in the exploration region, and the condition function, decreasing, indicating that the exploration condition worsens with time as a consequence of the area depletion. Three new methodologies are proposed using nonlinear control functions to explain the influence of technological progress in the reserves accrual. Acting as a proxy for exploratory effort, the drilling footage is used as an explanatory variable for both the control and the condition functions. The aforementioned methodologies were tested using a dataset of five petroliferous basins. After evaluating the explicative capacity by fitting the models to the historical data, out of sample forecast were made for a horizon of 3 and 10 years. The results using a dataset of four different basins indicate that the drilling footage can improve the long-term forecast. The analysis in the residues of the proposed models indicates that the models captured the information contained in the data and satisfactorily describes the process of evolution of discoveries in the observed series.

\section{Keywords}

Evolution of Discoveries, Recoverable Oil Volume, Petroleum Basin, Forecast. 


\section{Sumário}

1. Introdução 1

1.1. O problema 1

1.2. Objetivos 3

1.3. Motivação e justificativa 3

1.4. Contribuição 4

1.5. Apresentação dos capítulos 5

2. Metodologia de pesquisa $\quad 6$

2.1. Limitações 6

2.2. Abordagem da revisão bibliográfica 6

2.3. Validação analítica da bibliografia de referência

$\begin{array}{ll}\text { 2.4. Necessidade de novos modelos } & 7\end{array}$

2.5. Dados 8

2.6. Planejamento de experimentos 9

3. Revisão bibliográfica 11

3.1. O Método "Creaming" 12

3.1.1. Modelo de probabilidade de sucesso 13

3.1.2. Modelo de probabilidade de volume de campo 14

3.1.3. Predição do volume total para "m" futuros poços pioneiros 15

3.2. Métodos baseados no princípio da exaustão física 18

3.3. Metodologias propostas pelo USGS 21

3.3.1. Recursos não descobertos (descobertas de novos campos) 21

3.3.2. Crescimento de reservas dos campos existentes 23

3.4. O Pico de Hubbert 25

3.5. Metodologia baseada nas funções de controle e de condição 29

3.5.1. Modelos determinísticos: evolução com o tempo $(\mathrm{t})$

3.5.2. Modelos estocásticos: evolução com o tempo $(\mathrm{t})$

3.5.3. Modelos determinísticos: evolução com o volume de perfuração 37

3.6. Considerações 40

4. Metodologias propostas 42

4.1. Utilizando uma função de controle não linear 42 
4.2. Considerando novos objetivos exploratórios 50

4.3. Utilizando controle e condição em função do esforço exploratório 55

5. Análise de resíduos 58

5.1. A integral de correlação 59

5.2. A estatística BDS 62

5.3. Escolha de parâmetros $\quad 64$

6. Resultados dos ajustes "in sample" 65

6.1. Evolução das descobertas em função do volume do ano anterior 66

6.1.1. Metodologia I 66

6.1.1.1. Bacia de Campos 66

6.1.1.2. Bacia do Recôncavo 69

6.1.1.3. Bacia do Rio Grande do Norte/Ceará 72

6.1.1.4. Bacia de Sergipe/Alagoas $\quad 74$

6.1.1.5. Golfo do México 76

$\begin{array}{ll}\text { 6.1.2. Metodologia II } & 78\end{array}$

6.1.2.1. Bacia de Campos 78

6.1.2.2. Bacia do Recôncavo 80

6.1.2.3. Bacia do Rio Grande do Norte/Ceará 83

6.1.2.4. Bacia de Sergipe/Alagoas 85

6.1.2.5. Golfo do México 87

6.1.3. Metodologia III 89

6.1.3.1. Bacia do Recôncavo 89

6.2. Evolução das descobertas em função do volume de perfuração 99

6.2.1. Metodologia IV 99

6.2.1.1. Bacia de Campos 99

6.2.1.2. Bacia do Recôncavo 103

6.2.1.3. Bacia do Rio Grande do Norte/Ceará 105

6.2.1.4. Bacia do Sergipe/Alagoas 108

6.2.2. Metodologia V 111

6.2.2.1. Bacia de Campos 111

6.2.2.2. Bacia do Recôncavo 114

6.2.2.3. Bacia do Rio Grande do Norte/Ceará 117

$\begin{array}{ll}\text { 6.2.2.4. Bacia de Sergipe/Alagoas } & 120\end{array}$ 
7. Resultados das previsões "out of sample" 126

7.1. Previsões a curto prazo: modelos R(t) - metodologias I, II e III 126

$\begin{array}{ll}7.1 .1 . \text { Metodologia I } & 126\end{array}$

7.1.1.1. Bacia de Campos 127

7.1.1.2. Bacia do Recôncavo 127

7.1.1.3. Bacia do Rio Grande do Norte/Ceará 128

$\begin{array}{ll}\text { 7.1.1.4. Bacia de Sergipe/Alagoas } & 128\end{array}$

7.1.1.5. Golfo do México 129

$\begin{array}{ll}\text { 7.1.2. Metodologia II } & 130\end{array}$

$\begin{array}{ll}\text { 7.1.2.1. Bacia de Campos } & 130\end{array}$

7.1.2.2. Bacia do Recôncavo 130

7.1.2.3. Bacia do Rio Grande do Norte/Ceará 131

7.1.2.4. Bacia de Sergipe/Alagoas 132

7.1.2.5. Golfo do México 132

7.1.3. Metodologia III 133

7.1.3.1. Bacia do Recôncavo 133

7.2. Previsões a longo prazo: modelos $R(L)$ - metodologias IV e V 134

$\begin{array}{ll}\text { 7.2.1. Metodologia IV } & 134\end{array}$

7.2.1.1. Bacia de Campos 134

7.2.1.2. Bacia do Recôncavo 135

7.2.1.3. Bacia do Rio Grande do Norte/Ceará 136

$\begin{array}{ll}\text { 7.2.1.4. Bacia do Sergipe/Alagoas } & 137\end{array}$

$\begin{array}{ll}\text { 7.2.2. Metodologia } V & 138\end{array}$

$\begin{array}{ll}\text { 7.2.2.1. Bacia de Campos } & 138\end{array}$

7.2.2.2. Bacia do Recôncavo 139

7.2.2.3. Bacia do Rio Grande do Norte/Ceará 140

$\begin{array}{ll}\text { 7.2.2.4. Bacia de Sergipe/Alagoas } & 141\end{array}$

7.3. Análise das Previsões 144

7.3.1. Bacia de Campos 144

7.3.2. Bacia do Recôncavo 145

$\begin{array}{ll}\text { 7.3.3. Bacia do RN/CE } & 146\end{array}$

$\begin{array}{ll}\text { 7.3.4. Bacia de SE/AL } & 147\end{array}$

$\begin{array}{ll}\text { 7.3.5. Golfo do México } & 149\end{array}$ 
$\begin{array}{ll}\text { Referências bibliográficas } & 158\end{array}$

Bibliografia 163

Apêndice A: definições 167

Apêndice B: demonstração das fórmulas de Knoring et al. (1999) 171

Apêndice C: metodologia USGS para volumes não descobertos 198

Apêndice D: aplicações de crescimento de reservas 210

Apêndice E: simplificação dos modelos propostos 217

Apêndice F: equações no formato do E-Views 4.1 233

Apêndice G: resultados do Teste BDS 234

Apêndice $\mathrm{H}$ : análise dos dados do recôncavo 239 


\section{Lista de figuras}

Figura 1 - Previsão de descobertas pelo Método de Creaming. 12

Figura 2 - Curvas simétricas: Gaussiana, Lorentziana e Logística. $\quad 25$

Figura 3 - Produção anual dos EUA - Ajuste com curva Gaussiana. 26

Figura 4 - Produção anual mundial - Ajuste com Curvas Gaussianas. 26

Figura 5 - Produção e Descoberta acumulada (dados dos EUA):

Curva Gaussiana.

Figura 6 - Produção e Descoberta acumulada (dados mundiais):

Curva Gaussiana.

Figura 7 - Descobertas acumuladas vs. tempo: simetria em

relação ao ponto de inflexão.

Figura 8 - Descobertas acumuladas vs. tempo: assimetria em relação ao ponto de inflexão.

Figura 9 - Princípio da modelagem do acréscimo de descobertas $(\Delta R) .30$

Figura 10 - Diferentes formas da função seleção de condições $f(R)$. 33

Figura 11 - Influência da função seleção de condições no gráfico $(\Delta R) .33$

Figura 12 - Seleção de campos devido à restrição de investimentos. 34

Figura 13 - Modelagem dos Componentes Aleatórios:

$Z_{t}=R_{t}-P_{t}$ e $Z_{t}^{\prime}=\Delta R_{t}-\Delta P_{t}$.

Figura 14 - "k" muda de acordo com o aumento no volume de perfuração $(L)$.

Figura 15 - Forma específicas da função "k" divididas em 3 grupos.

Figura 16 - Influência do preço do petróleo nos resultados

subseqüentes e vice-versa.

Figura 17 - Acréscimo no volume $\left(R_{t}\right)$ devido ao progresso tecnológico.

Figura $18-\varphi(R)$ não linear. Grandes descobertas: a) no início; b) no final da exploração.

Figura 19 - Efeito cascata devido ao aumento no preço do petróleo.

Figura 20 - Curva de crescimento populacional.

Figura 21 - Curva de aprendizagem.

Figura 22 - Curva logística.

Figura 23 - Função Seleção de Condições considerando novos objetivos exploratórios.

Figura 24 - Função seleção de controle considerando novos objetivos exploratórios.

Figura 25 - Funções de controle e de condição propostas com grandes descobertas: a) no início; b) no final do processo exploratório. 52 
Figura 26 - Curva $\Delta \mathrm{R}$ proposta considerando novos objetivos exploratórios com grandes descobertas: a) no início; b) no final do processo exploratório.

Figura 27 - Controle e condição em função do volume exploratório (L). 55

Figura 28 - Região de aceitação e crítica do Teste BDS para o intervalo de confiança $95 \%$.

Figura 29 - Bacia de Campos: Gráfico R(t) vs. tempo. 67

Figura 30 - Bacia de Campos: Resultado do Modelo Knoring_8. 68

Figura 31 - Bacia de Campos: Função seleção de condições:

Modelo Knoring_8.

Figura 32 - Bacia do Recôncavo: Gráfico R(t) vs. tempo. 70

Figura 33 - Bacia do Recôncavo: Resultado do Modelo Knoring_7. 71

Figura 34 - Bacia do Recôncavo: Função seleção de condições:

Modelo Knoring_7.

Figura 35 - Bacia do RN/CE: Gráfico R(t) vs. tempo.

Figura 36 - Bacia do RN/CE: Resultado do Modelo Knoring_8.

Figura 37 - Bacia do RN/CE: Função seleção de condições:

Modelo Knoring_8.

Figura 38 - Bacia de SE/AL: Gráfico R(t) vs. tempo. 74

Figura 39 - Bacia de SE/AL: Resultado do Modelo Knoring_8. 75

Figura 40 - Bacia de SE/AL: Função seleção de condições:

Modelo Knoring_8.

Figura 41 - Golfo do México: Gráfico R(t) vs. tempo. 76

Figura 42 - Golfo do México: Resultado do Modelo Knoring_9. 77

Figura 43 - Golfo do México: Função seleção de condições:

Modelo Knoring_9.

Figura 44 - Bacia de Campos: Resultado do Modelo 6x7. 80

Figura 45 - Bacia do Recôncavo: Resultado do Modelo 7x8. 82

Figura 46 - Função seleção de controle e função seleção de condição: Modelo $7 \times 8$.

Figura 47 - Bacia do RN/CE: Resultado do Modelo 1x2. 85

Figura 48 - Bacia de SE/AL: Resultado do Modelo 7x9. 87

Figura 49 - Golfo do México: Resultado do Modelo 7x8. 89

Figura 50 - Curvas "S" no gráfico R(t) vs. tempo. 90

Figura 51 - Distribuição dos campos de óleo e gás na Bacia do Recôncavo.

Figura 52 - Seção geológica esquemática do campo de Riacho da Barra. 
Figura 53 - Bacia do Recôncavo: Resultado do ajuste de curvas nos três intervalos sugeridos.

Figura 54 - Bacia de Campos: Número de poços acumulado (L) vs. tempo.

Figura 55 - Defasagem entre o esforço exploratório anual $(\Delta \mathrm{L})$

e o acréscimo de volume $(\Delta R)$.

Figura 56 - Bacia de Campos: Influência dos poços injetores nos acréscimos recuperáveis.

Figura 57 - Bacia de Campos: Resultado do Modelo L_9.

Figura 58 - Bacia de Campos: Eficiência da exploração - k(L) vs. L Modelos L_9.

Figura 59 - Bacia do Recôncavo: Número de poços acumulado (L) vs. tempo.

Figura 60 - Bacia do Recôncavo: Influência dos poços injetores nos acréscimos recuperáveis.

Figura 61 - Bacia do Recôncavo: Resultado do Modelo L_9.

Figura 62 - Bacia do Recôncavo: Eficiência da exploração - k(L) vs. L

- Modelo L_2.

Figura 63 - Bacia do RN/CE: Número de poços acumulado (L) vs. tempo.

Figura 64 - Bacia do RN/CE: Influência dos poços injetores nos acréscimos recuperáveis.

Figura 65 - Bacia do RN/CE: Resultado do Modelo L_9.

Figura 66 - Bacia do RN/CE: Eficiência da exploração - k(L) vs. L - Modelos L_9.

Figura 67 - Bacia de SE/AL: Número de poços acumulado (L) vs. tempo.

Figura 68 - Bacia de SE/AL: Influência dos poços injetores nos acréscimos recuperáveis.

Figura 69 - Bacia de SE/AL: Resultado do Modelo L_9.

Figura 70 - Bacia de SE/AL: Eficiência da exploração - k(L) vs. L

- Modelos L_9.

Figura 71 - Bacia de Campos: Resultado do Modelo 1x8.

Figura 72 - Função Seleção de Controle: Modelo 1x8.

Figura 73 - Bacia do Recôncavo: Resultado do Modelo 7x3.

Figura 74 - Função Seleção de Controle: Modelo 7x3.

Figura 75 - Bacia do RN/CE: Resultado do Modelo 1x8.

Figura 76 - Função Seleção de Controle: Modelo $1 \times 8$.

Figura 77 - Bacia do SE/AL: Resultado do Modelo 4x8. 
Figura 78 - Função Seleção de Controle: Modelo 4x8.

Figura 79 - Bacia de Campos: Previsão 3 anos utilizando o Modelo Knoring_8.

Figura 80 - Bacia do Recôncavo: Previsão 3 anos utilizando o Modelo Knoring_7.

Figura 81 - Bacia do RN/CE: Previsão 3 anos utilizando o Modelo Knoring_8.

Figura 82 - Bacia do SE/AL: Previsão 3 anos utilizando o Modelo Knoring_8.

Figura 83 - Golfo do México: Previsão 3 anos utilizando o Modelo Knoring_9.

Figura 84 - Bacia de Campos: Previsão 3 anos utilizando o Modelo $6 \times 7$.

Figura 85 - Bacia do Recôncavo: Previsão 3 anos utilizando o Modelo $7 \times 8$.

Figura 86 - Bacia do RN/CE: Previsão 3 anos utilizando o Modelo $1 \times 2$.

Figura 87 - Bacia de Sergipe/Alagoas: Previsão 3 anos utilizando o Modelo $7 \times 9$.

Figura 88 - Golfo do México: Previsão 3 anos utilizando o Modelo $7 \times 8$.

Figura 89 - Bacia do Recôncavo: Previsão 3 anos utilizando o Modelo $1 \times 2$.

Figura 90 - Bacia de Campos: Previsão 10 anos utilizando o Modelo L_9.

Figura 91 - Bacia do Recôncavo: Previsão 10 anos utilizando o Modelo L_2.

Figura 92 - Bacia do RN/CE: Previsão 10 anos utilizando o Modelo L_9.

Figura 93 - Bacia do SE/AL: Previsão 10 anos utilizando o Modelo L_9.

Figura 94 - Bacia de Campos: Previsão 10 anos utilizando o Modelo $1 \times 8$.

Figura 95 - Bacia do Recôncavo: Previsão 10 anos utilizando o Modelo $7 \times 3$.

Figura 96 - Bacia do RN/CE: Previsão 10 anos utilizando o Modelo $1 \times 8$.

Figura 97 - Bacia de SE/AL: Previsão 10 anos utilizando o Modelo $4 \times 8$.

Figura 98 - Bacia de SE/AL: Previsão 5 anos utilizando o Modelo $4 \times 8$. 
Figura 99 - Bacia de SE/AL: Previsão 5 anos utilizando o Modelo $1 \times 8$.

Figura 100 - Volume recuperável em quatro dos principais campos da Bacia de Campos.

Figura 101 - Volume recuperável na Bacia RN/CE: Reavaliação em 1994.

Figura 102 - Volume recuperável no Campo W. 148

Figura 103 - Volume recuperável no Campo Y. 148

Figura 104 - Sistema de classificação de recursos. 167

Figura 105 - Formulários dos dados de entrada USGS. 203

Figura 106 - Estimativa média dos volumes mundiais (BBOE). 206

Figura 107 - Sessão estratigráfica na Península Arábica. 207

Figura 108 - Três TPS no Iraque. 207

Figura 109 - Resultados estimados para a Bacia San Joaquin. 209

Figura 110 - CGF vs. anos desde a primeira produção na bacia Siberiana Ocidental. 214

Figura 111 - CGF vs. YSFP - Siberiana Ocidental. Ajuste de curva para um e dois segmentos.

Figura 112 - CGF vs. YSFP a) Volga-Ural; b) Volga-Ural (sem Romashkino); c) ajuste "a"; d) ajuste "b"; e) EUA onshore (Attanasi et al., 1999); f) Golfo do México (Lore et al., 1996). 


\section{Lista de tabelas}

Tabela 1 - Previsões do suprimento mundial de óleo.

Tabela 2 - Série histórica proposta composta por 10 observações.

59

Tabela 3 - Cálculo dos pares Its.

Tabela 4 - Cálculo das normas dos pares Its.

Tabela 5 - Valores da soma dos quadrados dos resíduos aplicando a primeira metodologia proposta por Knoring et al. (1999) aos dados da Bacia de Campos.

Tabela 6 - Teste BDS - Bacia de Campos: Resíduos do Modelo Knoring_8.

Tabela 7 - Valores da soma dos quadrados dos resíduos aplicando a primeira metodologia proposta por Knoring et al. (1999) aos dados da Bacia do Recôncavo.

Tabela 8 - Valores da soma dos quadrados dos resíduos aplicando a primeira metodologia proposta por Knoring et al. (1999) aos dados da Bacia do Rio Grande do Norte/Ceará.

Tabela 9 - Valores da soma dos quadrados dos resíduos aplicando a primeira metodologia proposta por Knoring et al. (1999) aos dados da Bacia de SE/AL.

Tabela 10 - Valores da soma dos quadrados dos resíduos aplicando a primeira metodologia proposta por Knoring et al. (1999) aos dados do Golfo do México.

Tabela 11 - Metodologia II - Campos: Resultados utilizando controle não linear.

Tabela 12 - Metodologia II - Recôncavo: Resultados utilizando controle não linear.

Tabela 13 - Metodologia II - RN/CE: Resultados utilizando controle não linear.

Tabela 14 - Metodologia II - SE/AL: Resultados utilizando controle não linear.

Tabela 15 - Metodologia II - Golfo do México: Resultados utilizando controle não linear.

Tabela 16 - Análise do volume adicionado nos três intervalos (Resumo do Apêndice H).

Tabela 17 - Metodologia III - Recôncavo: Resultados utilizando somente os 11 anos iniciais.

Tabela 18 - Metodologia III - Recôncavo: Resultados utilizando os anos 12-26.

Tabela 19 - Metodologia III - Recôncavo: Resultados utilizando os anos 27-39. 
Tabela 20 - Bacia de Campos: soma dos quadrados dos resíduos (SS) - Metodologia IV.

Tabela 21 - Bacia do Recôncavo: soma dos quadrados dos resíduos (SS) - Metodologia IV.

Tabela 22 - Bacia do RN/CE: soma dos quadrados dos resíduos (SS) - Metodologia IV.

Tabela 23 - Bacia do SE/AL: soma dos quadrados dos resíduos (SS) - Metodologia IV.

Tabela 24 - Metodologia V - Campos: Resultados dos modelos empíricos.

Tabela 25 - Metodologia V - Recôncavo: Resultados dos modelos empíricos.

Tabela 26 - Metodologia V - RN/CE: Resultados dos modelos empíricos.

Tabela 27 - Metodologia V - SE/AL: Resultados dos modelos empíricos.

Tabela 28 - Resumo das Metodologias - Capítulo 6.

Tabela 29 - Bacia de Campos: previsão 3 anos - Metodologia I.

Tabela 30 - Bacia do Recôncavo: previsão 3 anos - Metodologia I. 127

Tabela 31 - Bacia do RN/CE: previsão 3 anos - Metodologia I. 128

Tabela 32 - Bacia de SE/AL: previsão 3 anos - Metodologia I. 129

Tabela 33 - Golfo do México: previsão 3 anos - Metodologia I. 129

Tabela 34 - Bacia de Campos: previsão 3 anos - Metodologia II. 130

Tabela 35 - Bacia do Recôncavo: previsão 3 anos - Metodologia II. 131

Tabela 36 - Bacia do RN/CE: previsão 3 anos - Metodologia II. 131

Tabela 37 - Bacia de Sergipe/Alagoas: previsão 3 anos -

Metodologia II. 132

Tabela 38 - Golfo do México: previsão 3 anos - Metodologia II. 132

Tabela 39 - Bacia do Recôncavo: previsão 3 anos - Metodologia III. 133

Tabela 40 - Bacia de Campos: Previsão 10 anos - Metodologia IV. 135

Tabela 41 - Bacia do Recôncavo: Previsão 10 anos -

Metodologia IV.

Tabela 42 - Bacia do Rio Grande do Norte/Ceará: Previsão 10 anos

- Metodologia IV.

Tabela 43 - Bacia do Sergipe/Alagoas: Previsão 10 anos

- Metodologia IV.

Tabela 44 - Bacia de Campos: Previsão 10 anos - Metodologia V. 139

Tabela 45 - Bacia do Recôncavo: Previsão 10 anos - Metodologia V. 140

Tabela 46 - Bacia do RN/CE: Previsão 10 anos - Metodologia V. 
Tabela 47 - Bacia de SE/AL: Previsão 10 anos - Metodologia V. 142

Tabela 48 - Bacia de SE/AL: Previsão 5 anos - Modelo 4x8. 143

Tabela 49 - Bacia de SE/AL: Previsão 5 anos - Modelo 1x8. 143

Tabela 50 - Bacia do Recôncavo: análise do ano 2001.

Tabela 51 - Resumo das Metodologias - Capítulo 7.

Tabela 52 - Estimativa de recursos não descobertos para o Iraque. 208

Tabela 53 - Histórico de reservas totais na Bacia Siberiana

Ocidental, com relação à data de descobrimento e YSFP.

Tabela 54 - Teste BDS - Metodologia I - Bacia do Recôncavo:

Resíduos do Modelo Knoring_7.

Tabela 55 - Teste BDS - Metodologia I - Bacia do RN/CE:

Resíduos do Modelo Knoring_8.

Tabela 56 - Teste BDS - Metodologia I - Bacia de SE/AL:

Resíduos do Modelo Knoring_8.

Tabela 57 - Teste BDS - Metodologia I - Golfo do México:

Resíduos do Modelo Knoring_9.

Tabela 58 - Teste BDS - Metodologia II - Bacia de Campos:

Resíduos do Modelo 6x7.

Tabela 59 - Teste BDS - Metodologia II - Bacia do Recôncavo:

Resíduos do Modelo 7x8.

Tabela 60 - Teste BDS - Metodologia II - Bacia do RN/CE:

Resíduos do Modelo 1x2.

Tabela 61 - Teste BDS - Metodologia II - Bacia de SE/AL:

Resíduos do Modelo 7x9.

Tabela 62 - Teste BDS - Metodologia II - Golfo do México:

Resíduos do Modelo 7x8.

Tabela 63 - Metodologia III - Recôncavo: Teste BDS nos resíduos dos três ajustes propostos.

Tabela 64 - Teste BDS - Metodologia IV - Bacia de Campos:

Resíduos do Modelo L_9.

Tabela 65 - Teste BDS - Metodologia IV - Bacia do Recôncavo:

Resíduos do Modelo L_2.

Tabela 66 - Teste BDS - Metodologia IV - Bacia do RN/CE:

Resíduos do Modelo L_9.

Tabela 67 - Teste BDS - Metodologia IV - Bacia de SE/AL:

Resíduos do Modelo L_9.

Tabela 68 - Teste BDS - Metodologia V - Bacia de Campos:

Resíduos do Modelo 1x8.

Tabela 69 - Teste BDS - Metodologia V - Bacia do Recôncavo:

Resíduos do Modelo 7×3. 
Tabela 70 - Teste BDS - Metodologia V - Bacia do RN/CE: Resíduos do Modelo $1 \times 8$.

Tabela 71 - Teste BDS - Metodologia V - Bacia de SE/AL: Resíduos do Modelo 4x8. 


\section{Lista de abreviaturas e siglas}

AGF - Annual Growth Factor

ANP - Agência Nacional do Petróleo, Gás e Biocombustíveis

$\mathrm{AU}$ - Assessment Units

BBO - Bilhões de barris de óleo

Gb - Bilhões de barris de óleo

BBOE - Bilhões de barris de óleo equivalente

$\mathrm{CGF}_{\mathrm{n}}$ - Cumulative Growth Factor in the year " $\mathrm{n}$ "

DEI - Departamento de Engenharia Industrial

EOR - Enhanced oil recovery

EUA - Estados Unidos da América

EUR - Estimated Ultimate Recovery

$f(R)$ - Função seleção de condição

FR - Fator de recuperação

i.i.d. - Independentes e identicamente distribuídos

IOR - Improved oil recovery

$K(L)$ - Indicador de eficiência

L - Esforço exploratório, número de poços exploratórios

NGL - Natural Gas Liquid

OIP - Oil in-place

OPEC - Organization of the Petroleum Exporting Countries

Petrobras - Petróleo Brasileiro S.A.

$\mathrm{R}^{2}$ - Coeficiente de correlação ou $R$-squared

$\mathrm{R}_{\mathrm{t}}=$ Volume de Óleo Recuperável no ano " $\mathrm{t}$ "

SPE - Society of Petroleum Engineers 
SS - Soma dos quadrados dos resíduos

TPS - Total Petroleum System

US\$ - Dólar Americano

USGS - United States Geological Survey

WPC - World Petroleum Council

YSFP - Years since first production

$Z_{i+1}$ - Resíduo, erro ou diferença entre os valores reais e ajustados.

API - Grau API - American Petroleum Institute

$\varphi(R)$ - Função seleção de controle

$\Delta R_{t+1}=$ Acréscimo do volume recuperável entre os anos " $\mathrm{t}$ " $\mathrm{e} " \mathrm{t+1}$ " 